\title{
Right Atrial Giant Myxoma Occupying the Right Ventricular Cavity
}

\author{
Amanollah Heidari ${ }^{*}$, Mohammad Nourizadeh ${ }^{1},{\text { Mohammad Hossein Najafi }{ }^{1}, \text { Sara Nourizadeh }}^{2}$, \\ Najmeh Assadinia ${ }^{3}$
}

${ }^{1}$ Department of Cardiac Surgery, Faculty of Medicine, Ahvaz Jundishapur University of Medical Sciences, Ahvaz, Iran

2Department of Cell \& Molecular Biology, Faculty of Biological Sciences, Kharazmi University, Tehran, Iran

${ }^{3}$ Department of Pathology, Shahidbeheshti Medical University, Tehran, Iran

Email: *heidari_a@ajums.ac.ir

How to cite this paper: Heidari, A. Nourizadeh, M., Najafi, M.H., Nourizadeh, S. and Assadinia, N. (2018) Right Atrial Giant Myxoma Occupying the Right Ventricular Cavity. Case Reports in Clinical Medicine, 7, 476-481. https://doi.org/10.4236/crcm.2018.7804 $\underline{2}$

Received: June 10, 2018

Accepted: August 18, 2018

Published: August 21, 2018

Copyright (C) 2018 by authors and Scientific Research Publishing Inc. This work is licensed under the Creative Commons Attribution International License (CC BY 4.0).

http://creativecommons.org/licenses/by/ $4.0 /$

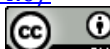

\section{Open Access}

\begin{abstract}
Introduction: Atrial myxomas are the most common primary heart tumors. Because of nonspecific symptoms, early diagnosis may be a challenge [1] [2]. Left atrial myxoma may or may not produce characteristic findings on auscultation. Two-dimensional echocardiography is the diagnostic procedure of choice. Most atrial myxomas are benign and can be removed by surgical resection. Cardiac myxoma is located mostly in left atrium [3]. This was amazing huge mass of cardiac myxoma in unusual part of the heart with the patient showed significant response to anticoagulation after presumed recurrence of tumor. Case Presentation: A case of giant right atrial myxoma mimicking the right ventricular tumor is described. Surgery was performed in 41 years old female and the fist like tumor with its stalk was excised. Surprisingly it recurred after 2 month as the smaller tumor was completely resolved with anticoagulation therapy. In addition because of previous normal echocardiography that was done for another reasons, we estimated the speed of tumor's growth (3 millimeter/month). Conclusion: To sum up a very large myxoma in right ventricle may only present with occasional dyspnea and we can diagnose it with precise evaluation and with performing on time echocardiography.
\end{abstract}

\section{Keywords}

Giant Myxoma, Right Atrial, Right Ventricular

\section{Introduction}

Intracadiac myxoma with an estimated incidence of $0.5-1$ per million per annum constitute about $60 \%$ of all primary heart tumor. Approximately $90 \%$ occur in the left atrial cavity, and the right atrium is an unusual location. Right atrial myxomas rarely produce symptoms until they are huge [4] [5]. Our case is of special interest because of its big size and the recurrence after 2 month. 
We had this opportunity to estimate tumor's growth rate because of serial normal echocardiograms that were performed for another non-cardiac reasons.

We presented this case to introduce the patient with atypical symptom of cardiac myxoma and unusual size and unusual response to treatment

\section{Case Presentation}

A 41 year-old woman complained of exertional dyspnea and cough 2 month prior to the admission. Electrocardiography was nonspecific but she had a systolic murmur in physical examination. A transthoracic 2D echocardiogram showed a large right atrial mass through the tricuspid valve (Figures 1-3). The contrast computerized tomographic scan showed an abnormal shadow defect in the right ventricle. The coronary angiography was normal. We diagnosed it as a right ventricular tumor.

The patient undergone surgery using Median sternotomy, after cardiac arrest by ante grade blood cardioplegia, a right atriotomy was done. Most of the mass was in the right ventricle of the tricuspid valve. The tumor was excised along with the stalk. Tricuspid valve repaired after that.

On gross inspection, the tumor was a soft, lobulated mass measuring $90 \times$ $20 \times 60 \mathrm{~mm}$, histological examination confirmed that it was a myxoma. A transthoracic echocardiogram obtained 5 days post operation that showed no residual tumor and mild tricuspid regurgitation. The patient discharged 5 days after surgery.

She came back 3 month after discharge with some dyspnea. Transthoracic echocardiogram revealed a mobile right atrial tumor measuring $10 \times 6 \mathrm{~mm}$.

Because the patient had nephrolithiasis and for this reason she had serial echo-study for preoperative evaluation the last normal trans-thoracic echocardiogram was 24 month ago (in 29/Jun/2011). The tumor was diagnosed in $19 / \mathrm{Jun} / 2013$. It's very striking finding that tumor in 24 month can grow up to the $90 \mathrm{~mm}$ length(the mean velocity of growth in length was at least 3.7 mm per month).

The patient rejected admission for another surgery so oral anticoagulation with warfarin started and close contact with patient preserved. Dyspnea gradually disappeared and repeated transthoracic echocardiogram after 2 month showed no tumor in the right atrium (Figure 4). The patient evaluated in 3 month by echocardiography and she was normal on 3 occasions.

\section{Discussion}

Primary cardiac neoplasms are rare and occur with an estimated incidence of $0.0017 \%$ to $0.19 \%$, representing less than $5 \%$ of all heart tumors [6] [7]. Myxoma is the most prevalent primary cardiac tumor. The RA is an unusual location and is the site of $15 \%$ to $20 \%$ of cases of myxoma. A low incidence of RA myxoma has been reported for decades in several series of autopsy cases. 
Approximately 70\% of affected patients are women [7] [8] predominantly between the third and sixth decades of life [8].

Myxomas are usually polypoid and pedunculated tumors (approximately $83 \%$ of cases) [8] [9]. In this report, our patient had a $90 \times 20 \times 60 \mathrm{~mm}$ a soft, lobulated mass.

In a recent publication reporting 19 years of experience with surgical treatment of primary intracardiac myxoma, seven $(17 \%)$ cases out of 41 originated from the RA [10]. However, in this case report, the mean maximal diameter of the tumors was measuring $90 \times 20 \times 60 \mathrm{~mm}$. To the best of our knowledge, our case is one of the largest RA myxomas described in the literature.

We report a case of a giant right atrial myxoma occupying the entire right ventricular cavity. Three aspects of this case are of special interest. First, the tumor in this patient is very large $90 \times 20 \times 60 \mathrm{~mm}$ that protruding in the right ventricle (Figure 5).

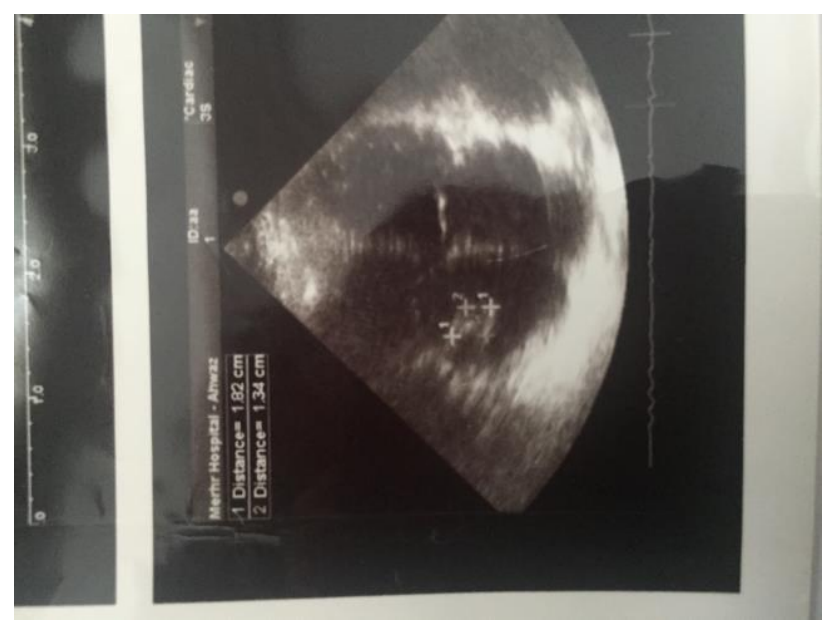

Figure 1. Myxoma in RA.

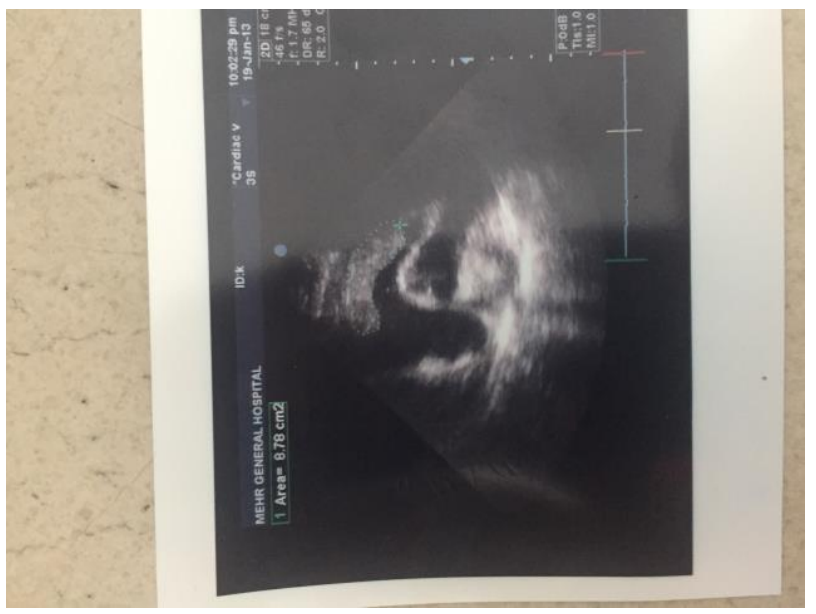

Figure 2. Extension of myxoma to RV. 

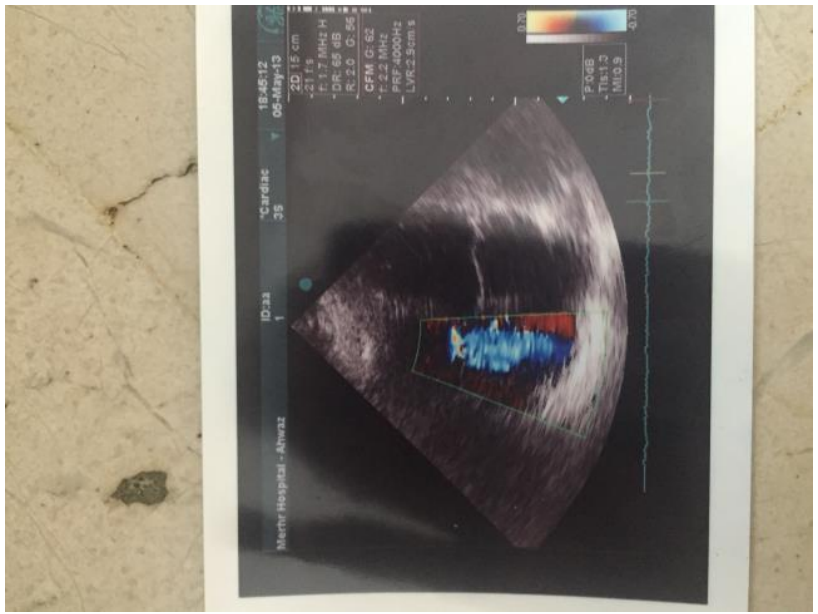

Figure 3. Tricuspid regurgitation due to myxoma.

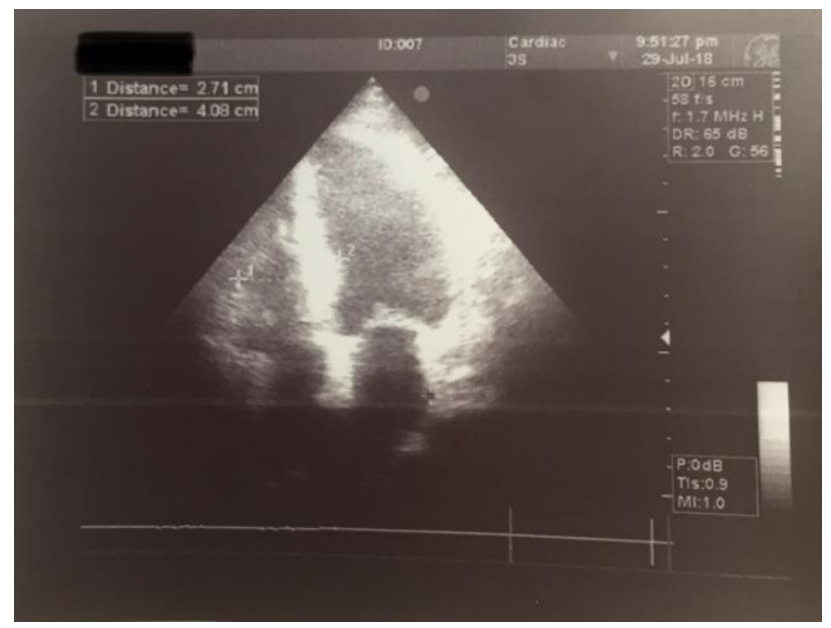

Figure 4. Myxoma is not seen in RA or RV.

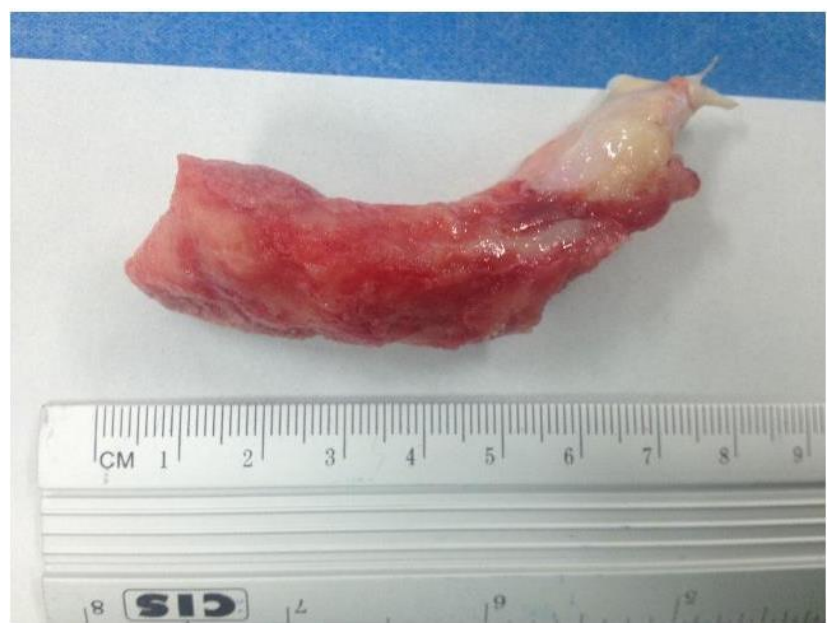

Figure 5. Size and shape of tumor in gross pathology.

Symptoms are said to be more variable when a tumor occurs in the right rather than the left atrium. It was, however, a striking feature of this patient's 
history, that the symptoms were not only vague but also mild and intermittent. Despite the presence of such a large tumor, one of the largest recorded, she could be free from symptoms for several weeks. Although this tumor is one of the largest myxoma that ever reported, the patients had only a mild dyspnea that it is possible that it was related to reductions in cardiac output to the pulmonary system produced by variable right atrial outflow obstruction. In comparison to the other myxoma this patients was diagnosed sooner because echocardiography performed in the first visit of patient.

Second patients come back with dyspnea again 3 moth after surgery. recurrence occurred in the site of previous tumor that successfully responds to oral anticoagulation therapy. because the shape of tumor is very similar to previous tumor in site of interatrial septum we wanted to excise it again but patient didn't accept surgery and she wanted to continue anticoagulation therapy with warfarin $5 \mathrm{mg}$ daily (The INR between 2 - 3) we surprisingly found that the tumor was resolved completely after 2 month and the patients be free of symptoms again.

So we recommended that if the myxoma like tumor recruited after the first surgery a trial of anticoagulation therapy performed before another surgery.

Third because the patients had normal echocardiography 24 month ago we had this opportunity that calculate the mean velocity of growth of the tumor

The first normal transthoracic echocardiogram was on 14//Jun/2008. Another normal one was on 29/Jun/2011. The first abnormal transthoracic echocardiogram was on 24/Jun/2013, 24 months after last normal echocardiogram. With a simple calculation we can conclude that linear growth of this tumor was at least approximately $3.7 \mathrm{~mm} /$ month. Previously reported myxoma have ranged in size from 1.5 to $11 \mathrm{~cm}$ [5]. although we didn't have serial echocardiography in these 2 years and the tumor may grow later, this calculation is the least mean velocity estimation and this is the first time that the mean velocity of the growth of myxoma tumor estimated.

\section{Conclusions}

In summary, this is the rare case report with large and rapid growing tumor in right side of the heart showing that initial intensive treatment with anticoagulation in patient with presumed recurrence of cardiac myxoma before secondary high risk surgery may result in a rapid clinical improvement and disappearing cardiac mass.

On the other hand if we assume another recurrence after previous surgery based on echocardiographic finding alone we should consider anticoagulation trial before performing another surgery.

\section{Acknowledgements}

The authors would like to thank Dr. Mohammad Hasan Adel who provided skillful editorial assistance. Financial support for editorial services was pro- 
vided by Ahwaz Jundishapur research center.

\section{Consent}

Written informed consent was obtained from the patient for publication of this case report and any accompanying images. A copy of the written consent is available for review by the Editor-in-Chief of this journal.

\section{Competing Interests}

The authors declare that they have no competing interests.

\section{Authors' Contributions}

All authors participated in the interpretation of clinical data and writing of this case report. All authors read and approved the final manuscript.

\section{References}

[1] Reynen, K. (1996) Frequnecy of Primary Tumor of the Heart. The American $\begin{array}{llll}\text { Journal of } & \text { Cardiology, } & 17, & 107 .\end{array}$ https://doi.org/10.1016/S0002-9149(97)89149-7

[2] Pavlides, G.S., Levin, R.N. and Hauser, A.M. (1989) Left Ventricular Recurrence of a Resected Left Atrial Myxoma. American Heart Journal, 117, 1390-1392. https://doi.org/10.1016/0002-8703(89)90427-4

[3] Fang, B.R., Chang, C.P., Cheng, C.W., et al. (2004) Tatal Detachment of Cardiac Myxoma Causing Saddle Embolization and Mimicking Aortic Dissection. Japanese Heart Journal, 45, 56-63.

[4] Bigelow, J.C., Herr, R.H. and Starr, A. (1969) Atrial Myxoma. Surgery, 65, 247-255.

[5] Bahnson, H.T. and Newman, E.V. (1953) Diagnosis and Surgical Removal of Intracavitary Myxoma of the Right Atrium. Bulletin of the Johns Hopkins Hospital, 93, 150-163.

[6] Guhathakurta, S. and Riordan, J.P. (2000) Surgical Treatment of Right Atrial Myxoma. Texas Heart Institute Journal, 27, 61-63.

[7] Azevedo, O., Almeida, J., Nolasco, T., Medeiros, R., Casanova, J., Bartosch, C., Almeida, J. and Pinho, P. (2010) Massive Right Atrial Myxoma Presenting as Syncope and Exertional Dyspnea: Case Report. Cardiovascular UItrasound, 8, 23. https://doi.org/10.1186/1476-7120-8-23

[8] Manfroi, W., Vieira, S.R., Saadi, E.K., Saadi, J. and Alboim, C. (2001) Multiple Recurrences of Cardiac Myxomas with Acute Tumoral Pulmonary Embolism [in Portuguese]. Arquivos Brasileiros de Cardiologia, 77, 161-163. https://doi.org/10.1590/S0066-782X2001000800007

[9] Ojji, D.B., Ajiduku, S.S., Omonua, O.O., Abdulkareem, L.L. and Parsonage, W. (2008) A Probable Right Atrial Myxoma Prolapsing through the Tricuspid Valve into the Right Ventricle: A Case Report. Cases Journal, 1, 386. https://doi.org/10.1186/1757-1626-1-386

[10] Samanidis, G., Perreas, K., Kalogris, P., Dimitriou, S., Balanika, M., Amanatidis, G., Khoury, M. and Michalis, A. (2011) Surgical Treatment of Primary Intracardiac Myxoma: 19 Years of Experience. Interactive CardioVascular and Thoracic Surgery, 13, 597-600. https://doi.org/10.1510/icvts.2011.278705 